\title{
Low-carbon unit commitment with intensive wind power generation and carbon capture power plant
}

\author{
Jiaming LI, Jinyu WEN (ه), Xingning HAN
}

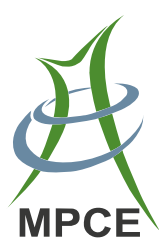

\begin{abstract}
The paper proposes a stochastic unit commitment (UC) model to realize the low-carbon operation requirement and cope with wind power prediction errors for power systems with intensive wind power and carbon capture power plant (CCPP). A linear re-dispatch strategy is introduced to compensate the wind power deviation from the spot forecast. The robust optimization technique is employed to obtain a reliable commitment plan against all realizations of wind power within the uncertainty set given by probabilistic forecast. The proposed model is validated with IEEE 39-bus system. The advantages of flexible CCPPs are compared to the normal coal-fueled plants and the impacts of robustness controlling are discussed.
\end{abstract}

Keywords Low-carbon unit commitment, Carbon capture and storage, Linear re-dispatch strategy, Robust optimization

\section{Introduction}

The global warming attracts more and more attentions in the recent few decades [1]. Relevant researches advocated that the excessive emission of greenhouse gases is the main cause [2]. As a result, the United Nations framework convention on climate change (UNFCCC) was proposed and Kyoto Protocol has been signed. According to the protocol, low-carbon

CrossCheck date: 18 December 2014

Received: 3 October 2014/ Accepted: 30 December 2014/Published online: 23 January 2015

(C) The Author(s) 2015. This article is published with open access at Springerlink.com

J. LI, J. WEN, X. HAN, State Key Laboratory of Advanced

Electromagnetic Engineering and Technology, School of

Electrical and Electronic Engineering, Huazhong University of

Science and Technology, Wuhan 430074, China

$(\bowtie)$ e-mail: jinyu.wen@hust.edu.cn development aiming to reduce $\mathrm{CO}_{2}$ emission is essential for the contracting parties including China. In 2005, an intergovernmental panel on climate change (IPCC) special report [3] showed that the electrical power generation accounts for over $34 \%$ of the global $\mathrm{CO}_{2}$ emissions owing to the popularity of fossil-fueled power plants. Hence, power system should be one of the main frontiers in the low-carbon revolution.

It can be noticed that there are two available solutions when it comes to low-carbon development in electric power generations. One is to control the $\mathrm{CO}_{2}$ emission in the existing fossil-fueled plant, while the other one is to seek for alternative clean power sources. Therefore, the carbon capture and storage (CCS) technique is applied to absorb and seal the $\mathrm{CO}_{2}$ produced by power plants while the renewable energy sources including wind and solar power are rapidly exploited throughout the world.

Undoubtedly, the integration of carbon capture power plant (CCPP) and renewable energy is bringing out new challenges and ideas to power system operation and dispatch. As for CCS technique, a mix-integer linear model for CCPP dispatch was proposed in [4], only considering the fixed $\mathrm{CO}_{2}$ capture rate. The potential of flexible control in CCS consumption power was reported to swiftly adjust the net output of power plant in [5]. In [6], typical methods in flexible CCS control were summarized and the corresponding model was proposed for flexible CCPP in steady-state operation analysis. In [7] and [8], the optimal strategies were discussed to maximize the operation profits from flexible CCPPs and the results showed that premium profits may be obtained by properly adjusting the $\mathrm{CO}_{2}$ capture rate according to the electricity and carbon prices. As for renewable energy, the focus is mainly on coping with the forecast errors. The scenarios, intervals, and risk index for the descriptions of wind power forecast errors were introduced in [9], and it is argued that forecast errors are inevitable and not negligible because of the stochastic nature of wind speed and the parameter uncertainties in the practical 
wind power curve. Consequently, the spinning reserves were adopted to cover the possible forecast deviations in [10, 11], but this modification in spinning reserves is theoretically proved to be inadequate in large deviation situations. Hence, several stochastic unit commitment (UC) models have been proposed, including scenarios-based optimization [12, 13], robust optimization with interval uncertainty $[14,15]$ and chance-constrained stochastic UC [16], to inherently utilize the different descriptions in wind power forecast uncertainties. All the literatures above are also suitable to involve the stochastic nature of other renewable resources.

After combining low-carbon technique and renewables integration, it is claimed that fossil-fueled plants equipped with flexible CCS devices possess higher ramping rate and lower minimum output, which is favorable for wind generation accommodation in [17, 18]. A UC model for power system with flexible CCPP and wind generation was proposed in [19], and the frequency response was considered in [20]. However, few literatures have discussed the effects on wind power forecast uncertainties, putting the system in unplanned operation status when the wind power deviation is significantly large.

This paper proposes a stochastic UC model considering intensive wind power generation and coal-fueled plants with flexible CCS devices. The economical operation with $\mathrm{CO}_{2}$ emission cost is set as the optimization target. A linear re-dispatch strategy is introduced to compensate the wind power deviations to cover the uncertainties. Robust optimization theory is thus applied to provide the reliable countermeasures for power system operators to deal with the wind power deviations.

The paper is organized as follows: Section 2 elaborates relevant issues in low-carbon unit commitment (LCUC) model. Section 3 presents the mathematical formulation of the proposed LCUC model. The transformation of LCUC model into a deterministic MILP problem based on linear robust optimization is presented in Section 4. Case studies in New England 39-bus system are illustrated in section 5 and the conclusions are given in section 6 .

\section{Relevant issues in LCUC}

\subsection{Characteristic of carbon capture power plant}

In this paper, the coal-fueled power plant equipped with post-combustion solvent/sorbent carbon capture system, which is the most commercially available and efficient type of CCS techniques nowadays, is considered [3]. The CCPP can flexibly control the $\mathrm{CO}_{2}$ capture rate along with its consumption power by venting channel or sorbent/ solvent storage tanks [6].

According to the previous studies, the output power of CCPPs can be written as follows [8]:
$P_{i, t}^{\mathrm{CCP}}=P_{i, t}^{\mathrm{Coal}}-P_{i, t}^{\mathrm{CCS}}$

where $P_{i, t}^{\mathrm{CCP}}$ is the total output of carbon capture plant; $P_{i, t}^{\text {Coal }}$ is the output of plant without CCS system.

$P_{i, t}^{\mathrm{CCS}}$ is the power required by CCS devices of CCPP $i$ in time $t$, which can be expressed as

$\left\{\begin{array}{l}P_{i, t}^{\mathrm{CCS}}=P_{i \mathrm{FI}}^{\mathrm{CCS}}+\lambda_{i}^{\mathrm{GE}} \beta_{i, t}^{\mathrm{CCS}} e_{i}^{\mathrm{G}} P_{i, t}^{\mathrm{Coal}} \\ \beta_{i \text { min }}^{\mathrm{CCS}} \leq \beta_{i, t}^{\mathrm{CCS}} \leq \beta_{i \max }^{\mathrm{CCS}}\end{array}\right.$

where $P_{i \mathrm{FIX}}^{\mathrm{CCP}}$ is the fixed power penalty independent from the operation state; $\lambda_{i}^{\mathrm{GE}}$ is the electric energy consumption to capture 1 ton of $\mathrm{CO}_{2} ; e_{i}^{\mathrm{G}}$ is the gross emission of $\mathrm{CO}_{2}$ when generating $1 \mathrm{MWh}$ of electric energy, and $\lambda_{i}^{\mathrm{GE}}$ is the adjustable $\mathrm{CO}_{2}$ capture rate $\left(\beta_{i, t}^{\mathrm{CCS}}\right.$ and $e_{i}^{\mathrm{G}}$ can be assumed as constants for a specific CCPP).

For simplicity, coal-fueled plant without CCS is not independently modeled in this paper, since it can be seen as a special CCPP when $\beta_{i \text { min }}^{\mathrm{CCS}}=\beta_{i \max }^{\mathrm{CCS}}=0$ and $P_{i \mathrm{FIX}}^{\mathrm{CCS}}=0$. In this paper, 'coal-fueled plant' only refers to the part of CCPP excludes CCS device.

\subsection{Description of wind power forecast error}

The stochastic nature of wind power leads to the poor accuracy in short-term spot forecast. Hence probabilistic approaches providing expected wind power values along with quantitative uncertainty description may be better choices.

As mentioned in Section 1, there are roughly three methods to quantify the forecast uncertainty. The most widely used one is the interval description, which gives the bounds of possible wind power under different confidence levels [9].

The wind power $W_{m, t}$ is expressed as

$\left\{\begin{array}{l}W_{m, t}=W_{m, t}^{\mathrm{F}}+\Delta W_{m, t} \\ \underline{W}_{m, t} \leq W_{m, t} \leq \bar{W}_{m, t}\end{array}\right.$

where $W_{m, t}^{\mathrm{F}}$ is the expected wind output of plant $m$ at time $t$; $\bar{W}_{m, t}$ is the upper limit; $\underline{W}_{m, t}$ is the lower limit; $\Delta W_{m, t}$ is the power deviation from $W_{m, t}^{\mathrm{F}}$, which is unknown in day-ahead UC planning. Thus, $W_{m, t}$ should be treated as a random variable.

\subsection{Wind power curtailment}

The possible wind power curtailment is always considered as an economical choice or a measure to control exceeded range of wind power fluctuations. In this paper, the curtailment power $W_{m, t}^{\text {Cur }}$ in the UC model is an uncertain variable defined by a given ratio $\mu_{m, t}^{\mathrm{W}}$ of the available wind power as follows. 
$\left\{\begin{array}{l}W_{m, t}^{\mathrm{Cur}}=\mu_{m, t}^{\mathrm{W}} W_{m, t} \\ 0 \leq \mu_{m, t}^{\mathrm{W}} \leq 1\end{array}\right.$

\subsection{Linear re-dispatch strategy}

The deviation of wind power from spot forecast value must be compensated by other power plants to maintain normal frequency. In this paper, a linear re-dispatch strategy is proposed to fulfill this power regulation, and it can be expressed as

$$
\begin{aligned}
& \left\{\begin{array}{l}
\Delta P_{i, m, t}^{\mathrm{Coal}}=K_{i, m, t}^{\mathrm{Coal}} \Delta W_{m, t} \\
\Delta P_{i, m, t}^{\mathrm{CCS}}=K_{i, m, t}^{\mathrm{CCS}} \Delta W_{m, t}
\end{array}\right. \\
& \sum_{i \in G}\left(\Delta P_{i, m, t}^{\mathrm{Coal}}+\Delta P_{i, m, t}^{\mathrm{CCS}}\right)+\left(1-\mu_{m, t}^{\mathrm{W}}\right) \Delta W_{m, t}=0
\end{aligned}
$$

where $K_{i, m, t}^{\mathrm{Coal}}$ and $K_{i, m, t}^{\mathrm{CCS}}$ are the coefficients with regard to the output adjustment in CCP $i$ to compensate the output deviation of wind plant $m ; \Delta P_{i, m, t}^{\text {Coal }}$ and $\Delta P_{i, m, t}^{\mathrm{CCS}}$ are the power adjustment conducted by both the coal-fueled plant and the CCS device; $K_{i, m, t}^{\mathrm{Coal}}$ and $K_{i, m, t}^{\mathrm{CCS}}$ are variables determined by solving the UC problem; $G$ is the set of CCPPs.

Practically, small but fast changing power unbalance may still exist after re-dispatching and it is hard to predict or manually handle. As a result, AGC and primary frequency response will step in, but it is beyond the concern of UC model in this paper.

\section{$2.5 \mathrm{UC}$ robustness}

A robust UC means a day-ahead commitment plan that can provide feasible regions for real-time dispatching under all possible scenarios of wind power series. In this paper, it is equivalent to a specific unit commitment with the corresponding re-dispatching plan which is suitable for any wind power values within the uncertainty interval.

Usually the wind power is hardly to realize the extreme value at the same time in all the wind plants. Hence, $\Gamma_{t}^{\mathrm{W}}$ is introduced to restrict the simultaneous deviations of different random variables from the expected values as follows:

$$
\begin{gathered}
\underline{W}_{m, t}^{\Gamma} \leq W_{m, t} \leq \bar{W}_{m, t}^{\Gamma} \\
\left\{\begin{array}{l}
\underline{W}_{m, t}^{\Gamma}=W_{m, t}^{\mathrm{F}}-\varphi_{m, t}\left(W_{m, t}^{\mathrm{F}}-\underline{W}_{m, t}\right) \\
\bar{W}_{m, t}^{\Gamma}=W_{m, t}^{\mathrm{F}}+\varphi_{m, t}\left(\bar{W}_{m, t}-W_{m, t}^{\mathrm{F}}\right) \\
\sum_{m \in W} \varphi_{m, t} \leq \Gamma_{t}^{\mathrm{W}},|W| \geq \Gamma_{t}^{\mathrm{W}} \geq 0
\end{array}\right.
\end{gathered}
$$

where $W$ is the set of wind plants; $|W|$ is the number of wind plants in the power system; $\varphi_{m, t}$ is the decisive variable controlling the variation range of wind plant output according to $\Gamma_{t}^{\mathrm{W}}$. Under such an assumption, the actual limits of wind power $W_{m, t}$ are decisive variables to be optimized, represented by $\Gamma$-limits as $\bar{W}_{m, t}^{\Gamma}$ and $\underline{W}_{m, t}^{\Gamma}$.

\section{Low-carbon unit commitment model}

\subsection{Optimization objective}

The optimization objective of LCUC is to minimize the total cost for daily fuel consumption and $\mathrm{CO}_{2}$ emission under expected wind power output.

$$
\begin{aligned}
& \min \sum_{t}^{T_{\mathrm{UC}}}\left(C_{\mathrm{CO}_{2}} \sum_{i \in G} E_{i, t}^{\mathrm{CO}_{2}}+C_{\mathrm{Coal}} \sum_{i \in G} F_{i, t}^{\mathrm{Coal}}+\sum_{i \in G} C_{i, t}^{\mathrm{SU}}\right. \\
& \left.\quad+\sum_{i \in G} C_{i, t}^{\mathrm{SD}}\right)\left.\right|_{W_{m, t}=W_{m, t}^{\mathrm{F}}}
\end{aligned}
$$

where $C_{\mathrm{CO}_{2}}$ is the $\mathrm{CO}_{2}$ emission price; $C_{\mathrm{Coal}}$ is the coal price; $E_{i, t}^{\mathrm{CO}_{2}}$ is the $\mathrm{CO}_{2}$ equivalent emission; $F_{i, t}^{\mathrm{Coal}}$ is the coal consumption; $C_{i, t}^{\mathrm{SU}}$ and $C_{i, t}^{\mathrm{SD}}$ are the startup and shut down prices for CCP $i$ at time $t$.

\subsection{Constraints}

The following constraints are included in the LCUC model.

\section{1) Power balance}

The power balance should always be maintained between generations and load requirements.

$\sum_{i \in G}\left(P_{i, t}^{\mathrm{Coal}}-P_{i, t}^{\mathrm{CCS}}\right)+\sum_{m \in W}\left(W_{m, t}-W_{m, t}^{\mathrm{Cur}}\right)=\sum_{k \in L} P_{k, t}^{\mathrm{Load}}$

where $P_{k, t}^{\mathrm{Load}}$ is the power consumption of load $k$ at time $t$; $L$ is the set of loads.

\section{2) Spinning reserve}

The online generators are available to cover load prediction errors as well as possible power shortage induced by forced-tripping of generators.

$$
\left\{\begin{array}{l}
\sum_{i \in G}\left(\bar{P}_{i, t}^{\mathrm{Coal}}-\underline{P}_{i, t}^{\mathrm{CCS}}\right)+\sum_{m \in W}\left(W_{m, t}-W_{m, t}^{\mathrm{Cur}}\right) \geq \sum_{k \in L} P_{k, t}^{\mathrm{Load}}+\alpha_{t}^{\mathrm{up}} \\
\sum_{i \in G}\left(\underline{P}_{i, t}^{\mathrm{Coal}}-\bar{P}_{i, t}^{\mathrm{CCS}}\right)+\sum_{m \in W}\left(W_{m, t}-W_{m, t}^{\mathrm{Cur}}\right) \leq \sum_{k \in L} P_{k, t}^{\mathrm{Load}}-\alpha_{t}^{\mathrm{dn}}
\end{array}\right.
$$

where $\bar{P}_{i, t}^{\text {Coal }}$ and $\bar{P}_{i, t}^{\mathrm{CCS}}$ are the currently available upper power limits of coal-fueled plant and CCS system, respectively; $\underline{P}_{i, t}^{\mathrm{Coal}}$ and $\underline{P}_{i, t}^{\mathrm{CCS}}$ are the corresponding lower power limits; $\alpha_{t}^{\text {up }}$ and $\alpha_{t}^{\mathrm{dn}}$ are the upward and downward spinning reserve requirements. 
3) Coal-fueled plant output range

The power output range of coal-fueled plant is decided by (10)-(12), which correspond to the minimum and maximum technical power limits, online ramping limits and power limits for startup or shut down operations respectively.

$$
\left\{\begin{array}{l}
\underline{P}_{i, t}^{\text {Coal }} \leq P_{i, t}^{\text {Coal }} \leq \bar{P}_{i, t}^{\text {Coal }} \\
\bar{P}_{i, t}^{\text {Coal }} \leq u_{i, t} P_{i \text { max }}^{\text {Coal }} \\
u_{i, t} P_{i \text { min }}^{\text {Coal }} \leq P_{i, t}^{\text {Coal }}
\end{array}\right.
$$

where binary decisive variable $u_{i, t}$ is the status of CCPP $i$ at time $t$, CCPP is online when $u_{i, t}=1$ while CCPP is offline when $u_{i, t}=0 ; P_{i \max }^{\mathrm{Coal}}$ and $P_{i \text { min }}^{\mathrm{Coa}}$ are the technical maximum and minimum power limits.

$\left\{\begin{array}{l}\bar{P}_{i, t}^{\text {Coal }} \leq P_{i, t-1}^{\text {Coal }}+U_{i}^{\text {Coal }}+M_{i}^{\text {Coal }}\left(1-u_{i, t-1}\right)+M_{i}^{\text {Coal }}\left(1-u_{i, t}\right) \\ P_{i, t}^{\text {Coal }} \geq P_{i, t-1}^{\text {Coal }}-D_{i}^{\text {Coal }}-M_{i}^{\text {Coal }}\left(1-u_{i, t-1}\right)-M_{i}^{\text {Coal }}\left(1-u_{i, t}\right)\end{array}\right.$

where $U_{i}^{\text {Coal }}$ and $D_{i}^{\text {Coal }}$ are the maximum upward and downward ramping power of coal-fueled plant, respectively. $M_{i}^{\text {Coal }}=P_{i \text { max }}^{\text {Coal }}+U_{i, t}^{\text {Coal }}+D_{i, t}^{\text {Coal }} \quad$ is a large number required to disable these constraints when the generator is starting up or shutting down.

$\left\{\begin{array}{l}\bar{P}_{i, t}^{\mathrm{Coal}} \leq P_{i \mathrm{SU}}^{\mathrm{Coal}}+M_{i}^{\mathrm{Coal}} u_{i, t-1} \\ \bar{P}_{i, t}^{\mathrm{Coal}} \leq P_{i \mathrm{SD}}^{\mathrm{Coal}}+M_{i}^{\mathrm{Coal}} u_{i, t+1}\end{array}\right.$

where $P_{i}^{\mathrm{Co} \text { SU }}$ and $P_{i \mathrm{SD}}^{\mathrm{Coal}}$ are the maximum power right after startup and before shutting down, respectively.

\section{4) CCS consumption power range}

The consumption power range for CCS is identical to those of the coal-fueled plants as

$$
\begin{aligned}
& \left\{\begin{array}{l}
\underline{P}_{i, t}^{\mathrm{CCS}} \leq P_{i, t}^{\mathrm{CCS}} \leq \bar{P}_{i, t}^{\mathrm{CCS}} \\
\bar{P}_{i, t}^{\mathrm{CCS}} \leq u_{i, t} P_{i \mathrm{FIX}}^{\mathrm{CCS}}+\lambda_{i}^{\mathrm{GE}} \beta_{i \max }^{\mathrm{CCS}} e_{i}^{\mathrm{G}} P_{i, t}^{\mathrm{Coal}} \\
u_{i, t} P_{i \mathrm{FIX}}^{\mathrm{CCS}}+\lambda_{i}^{\mathrm{GE}} \beta_{i \min }^{\mathrm{CCS}} e_{i}^{\mathrm{G}} P_{i, t}^{\mathrm{Coal}} \leq \underline{P}_{i, t}^{\mathrm{CCS}}
\end{array}\right. \\
& \left\{\begin{array}{l}
\bar{P}_{i, t}^{\mathrm{CCS}} \leq P_{i, t-1}^{\mathrm{CCS}}+U_{i}^{\mathrm{CCS}}+M_{i}^{\mathrm{CCS}}\left(1-u_{i, t-1}\right)+M_{i}^{\mathrm{CCS}}\left(1-u_{i, t}\right) \\
P_{i, t}^{\mathrm{CCS}} \geq P_{i, t-1}^{\mathrm{CCS}}-D_{i}^{\mathrm{CCS}}-M_{i}^{\mathrm{CCS}}\left(1-u_{i, t-1}\right)-M_{i}^{\mathrm{CCS}}\left(1-u_{i, t}\right)
\end{array}\right.
\end{aligned}
$$

$$
\left\{\begin{array}{l}
\bar{P}_{i, t}^{\mathrm{CCS}} \leq P_{i \mathrm{SU}}^{\mathrm{CCS}}+M_{i} u_{i, t-1} \\
\bar{P}_{i, t}^{\mathrm{CCS}} \leq P_{i \mathrm{SD}}^{\mathrm{CCS}}+M_{i} u_{i, t+1}
\end{array}\right.
$$

where $U_{i}^{\mathrm{CCS}}$ and $D_{i}^{\mathrm{CCS}}$ are the maximum upward and downward power change of CCS, respectively. $M_{i}^{\text {CCS }}$ is a large number just like $M_{i}^{\mathrm{Coal}}$ and $M_{i}^{\mathrm{CCS}}=P_{i \mathrm{FIX}}^{\mathrm{CCS}}+\lambda_{i}^{\mathrm{GE}}$ $\beta_{i \max }^{\mathrm{CCS}} e_{i}^{\mathrm{G}} P_{i \max }^{\mathrm{Coal}}+U_{i, t}^{\mathrm{Coal}}+D_{i, t}^{\mathrm{Coal}}$.
5) Minimum up and down time constraint

For coal-fueled plants, it is impossible to restart the generators immediately after shutting down, or vice versa. The status of generators to consider the minimum up and down time constraints is restricted as

$$
\begin{aligned}
& \left\{\begin{array}{l}
u_{i, t}=1, t \leq T_{i \mathrm{on}}^{\mathrm{For}} \\
u_{i, t}=0, t \leq T_{i \mathrm{off}}^{\mathrm{For}}
\end{array}\right. \\
& \begin{array}{c}
1-\left(u_{i, t}-u_{i, t-1}\right) \geq u_{i, k} \quad \forall t>T_{i \mathrm{off}}^{\mathrm{For}}, \\
k=t-T_{i \mathrm{UP}}+1, \ldots, t-1
\end{array} \\
& u_{i, k} \geq u_{i, t-1}-u_{i, t} \quad \forall t>T_{i \text { on }}^{\mathrm{For}}, \\
& k=t-T_{i \mathrm{DN}}+1, \ldots, t-1
\end{aligned}
$$

where $T_{i \text { on }}^{\text {For }}$ and $T_{i \text { off }}^{\text {For }}$ are the time period to compel CCPP $i$ to keep online or offline at the beginning of commitment plan; $T_{i \mathrm{UP}}$ and $T_{i \mathrm{DN}}$ are the minimum up and down time, respectively.

6) Wind curtailment percentage

The wind curtailment percentage can be expressed as

$\sum_{t}^{T_{\mathrm{UC}}} W_{m, t}^{\mathrm{Cur}} \leq \rho_{m}^{\mathrm{Wcur}} \sum_{t}^{T_{\mathrm{UC}}} W_{m, t}$

where $\rho_{m}^{\mathrm{Wcur}}$ is the maximum percentage of daily wind energy curtailment for wind farm $m$ within any possible wind power scenarios.

7) $\mathrm{CO}_{2}$ emission

The emission of $\mathrm{CO}_{2}$ constraint is an optional requirement, expressed as

$\left\{\begin{array}{l}E_{i, t}^{\mathrm{CO}_{2}}=e_{i}^{\mathrm{G}} P_{i, t}^{\mathrm{Coal}}-\left(P_{i, t}^{\mathrm{CCS}}-u_{i, t} P_{i \mathrm{FIX}}^{\mathrm{CCS}}\right) / \lambda_{i}^{\mathrm{GE}} \\ \sum_{t}^{T_{\mathrm{UC}}} \sum_{i \in G} E_{i, t}^{\mathrm{CO}_{2}} \leq E_{\mathrm{CO}_{2}}^{\mathrm{max}}\end{array}\right.$

where $E_{\mathrm{CO}_{2}}^{\max }$ is the maximum $\mathrm{CO}_{2}$ emission for a whole day.

8) Transmission capacity

DC power flow model is introduced here to represent the limits of power flow in all transmission lines.

$\left\{\begin{array}{l}f_{h}=\sum_{r \in N} B_{r h}\left[\sum_{i \in G(r)}\left(P_{i, t}^{\mathrm{Coal}}-P_{i, t}^{\mathrm{CCS}}\right)+\sum_{m \in W(r)}\left(W_{m, t}-W_{m, t}^{\mathrm{Cur}}\right)-\sum_{k \in L(r)} P_{k, t}^{\mathrm{Load}}\right] \\ -\bar{f}_{h} \leq f_{h} \leq \bar{f}_{h}\end{array}\right.$

where $\bar{f}_{h}$ is the capacity of transmission line $h ; B_{r h}$ is the sensitivity between injection power into bus $r$ and power flow over line $h ; G(r), W(r)$ and $L(r)$ are the sets of CCPPs, wind plants and loads connected to bus $r$.

In summary, the proposed LCUC model consists of the optimization objective (7) and regular constraints (8)-(21) along with key constraints (2)-(6) mentioned above. 


\section{Linearization and robust counterparts}

\subsection{Linearization}

The original LCUC model has an optimization objective with non-linear cost of CCPP fuel consumption. Hence, the approximate linearization of the CCPP fuel cost by piecewise functions [21] is performed to apply linear robust optimization theory and to seek for better efficiency when solving the problem.

\subsection{Robust counterpart}

The linearized LCUC problem still contains lots of random variables introduced by wind power uncertainty, which is impossible to be solved directly. Hence it must be transformed into an equivalent deterministic form by duality theory, commonly called robust counterpart [22, 23].

The robust counterpart of (22) was presented in [23] with $a_{v}$ as the random coefficients bearing known bounds $a_{v}^{\mathrm{L}}, a_{v}^{\mathrm{U}}$ and expected values $\bar{a}_{v}$. Decisive variables $\varphi_{v}$ and $\Gamma$ are introduced just as (6) to control the variation range of $a_{v}$. It has been proved that (22) with $a_{v}$ in a restricted variation range as (23) is equivalent to robust counterpart (24), which has no random coefficients any more. $z$ and $p_{v}$ are dual variables without physical meanings.

$$
\begin{aligned}
& \forall \sum_{v} a_{v} x_{v} \leq b, \quad a_{v} \in\left[a_{v}^{\mathrm{L}}, \quad a_{v}^{\mathrm{U}}\right], \quad E\left(a_{v}\right)=\bar{a}_{v} \\
& \left\{\begin{array}{l}
\Re(\Gamma)=\bar{a}_{v}-\varphi_{v}\left(\bar{a}_{v}-a_{v}^{\mathrm{L}}\right) \leq a_{v} \leq \bar{a}_{v}+\varphi_{v}\left(a_{v}^{\mathrm{U}}-\bar{a}_{v}\right) \\
0 \leq \varphi_{v} \leq 1, \sum_{v} \varphi_{v} \leq \Gamma
\end{array}\right.
\end{aligned}
$$

Since reference [23] gives that $t_{v}^{\mathrm{F}}=a_{v}^{\mathrm{U}}-\bar{a}_{v}$ and $t_{v}^{\mathrm{F}}=\bar{a}_{v}-a_{v}^{\mathrm{L}}$

$$
\left\{\begin{array}{l}
\sum_{v} \bar{a}_{v} x_{v}+\Gamma z+\sum_{v} p_{v} \leq b \\
z+p_{v} \geq \max \left[\left(a_{v}^{\mathrm{U}}-\bar{a}_{v}\right) x_{v},-\left(\bar{a}_{v}-a_{v}^{\mathrm{L}}\right) x_{v}\right] \quad z \geq 0, p_{v} \geq 0
\end{array}\right.
$$

$$
\left\{\begin{array}{l}
W_{m, t}=W_{m, t}^{\mathrm{F}}+\Delta W_{m, t} \\
W_{m, t}^{\mathrm{Cur}}=\mu_{m, t}^{\mathrm{W}} W_{m, t}^{\mathrm{F}}+\mu_{m, t}^{\mathrm{W}} \Delta W_{m, t} \\
P_{i, t}^{\mathrm{Coal}}=P_{i, t}^{\mathrm{F}, \mathrm{Coal}}+\sum_{m \in W} K_{i, m, t}^{\mathrm{Coal}} \Delta W_{m, t} \\
P_{i, t}^{\mathrm{CCS}}=P_{i, t}^{\mathrm{F}, \mathrm{CCS}}+\sum_{m \in W}^{m} K_{i, m, t}^{\mathrm{CCS}} \Delta W_{m, t}
\end{array}\right.
$$

Before applying this robust counterpart transformation in the LCUC model, all the random power output must be rewritten as (25).

Then power balance constraint is converted without further transformation as

$\sum_{i \in G}\left(P_{i, t}^{\mathrm{F}, \mathrm{Coal}}-P_{i, t}^{\mathrm{F}, \mathrm{CCS}}\right)+\sum_{m \in W}\left(1-\mu_{m, t}^{\mathrm{W}}\right) W_{m, t}^{\mathrm{F}}=\sum_{k \in L} P_{k, t}^{\mathrm{Load}}$
However, (9)-(11), (13)-(14), (19) and (21) are still required to be transformed for solution. The upward spinning reserve constraint in (9) is demonstrated as

$$
\begin{aligned}
& -\sum_{i \in G}\left(\bar{P}_{i, t}^{\mathrm{Coal}}-\underline{P}_{i, t}^{\mathrm{CCS}}\right)-\sum_{m \in W} W_{m, t}^{\mathrm{F}}\left(1-\mu_{m, t}^{\mathrm{W}}\right) \\
& -\sum_{m \in W} \Delta W_{m, t}^{\mathrm{Cur}}\left(1-\mu_{m, t}^{\mathrm{W}}\right) \leq-\sum_{k \in L} P_{k, t}^{\mathrm{Load}}-\alpha_{t}^{\mathrm{up}}
\end{aligned}
$$

Secondly, the controllable variation range of random variables $\Delta W_{m, t}$ are expressed as

$$
\left\{\begin{array}{l}
-\varphi_{m, t}\left(W_{m, t}^{\mathrm{F}}-\underline{W}_{m, t}\right) \leq \Delta W_{m, t} \leq \varphi_{m, t}\left(\bar{W}_{m, t}-W_{m, t}^{\mathrm{F}}\right) \\
0 \leq \varphi_{m, t} \leq 1, \sum_{m \in W} \varphi_{m, t} \leq \Gamma_{t}^{\mathrm{W}}
\end{array}\right.
$$

Finally, robust counterpart according to (22)-(24) can be written as

$$
\begin{aligned}
& \sum_{i \in G}\left(\bar{P}_{i, t}^{\mathrm{Coal}}-\underline{P}_{i, t}^{\mathrm{CCS}}\right)-\Gamma_{t}^{W} z_{t}^{\mathrm{USR}}-\sum_{m \in W} p_{m, t}^{\mathrm{USR}} \geq \sum_{k \in L} P_{k, t}^{\mathrm{Load}}+\alpha_{t}^{\mathrm{up}} \\
& \left\{\begin{array}{l}
z_{t}^{\mathrm{USR}}+p_{m, t}^{\mathrm{USR}} \geq-\left(1-\mu_{m, t}^{\mathrm{W}}\right)\left(\bar{W}_{m, t}-W_{m, t}^{\mathrm{F}}\right) \\
z_{t}^{\mathrm{USR}}+p_{m, t}^{\mathrm{USR}} \geq\left(1-\mu_{m, t}^{\mathrm{W}}\right)\left(W_{m, t}^{\mathrm{F}}-\underline{W}_{m, t}\right) \\
z_{t}^{\mathrm{USR}} \geq 0, p_{m, t}^{\mathrm{USR}} \geq 0
\end{array}\right.
\end{aligned}
$$

The transformations of other constraints are similar so as to neglect the details in this paper. The LCUC problem is eventually changed into a MILP representation which is ready to be efficiently solved.

\section{Case study}

The case studies are conducted based on the modified New England 39-bus system. The generator and CCS data are listed in Table 1 and Table 2. In all the cases, three $800 \mathrm{MW}$ wind farms are connected to the bus 30, 32 and 39 , respectively. The forecast error for wind power predictions are assumed to be $30 \%$ while the curtailment limit $\rho_{m}^{\text {Wcur }}$ for each wind farm is set to be $1 \%$. The branch and base load data are adopted from 'case39' in Matpower 4.1 toolbox [24] for MATLAB. IBM ILOG CPLEX V12.4 is used as the MILP solver.

The load and wind farm output forecasts are given in per unit value in Fig. 1.

For simplicity, loads at different nodes share the same per unit forecast data. Finally, the price of $\mathrm{CO}_{2}$ emission is $30 \$ / \mathrm{tCO}_{2}$; the $\mathrm{CO}_{2}$ emission limit of a whole day is $100000 \mathrm{tCO}_{2}$; the spinning reserves $\alpha_{t}^{\text {up }}$ and $\alpha_{t}^{\mathrm{dn}}$ are set as $10 \%$ of the total loads and $\Gamma_{t}^{\mathrm{W}}$ is set to be 3 which leads to the most robust solution which means maximum ranges of wind power variations are considered. 


\subsection{Validation of LCUC model}

Fig. 2 indicates that the available generation range is able to cover the net load variation including the wind power forecast errors and spinning reserves.

The maximum load-carrying rates of all branches are obtained with wind power scenarios generated within the forecast intervals by Monte Carlo simulation. In Fig. 3, the maximum load-carrying rates are normalized by the transmission capacities. It can be noticed that all the branch loads are kept in a safe range.

If all the loads are served by the coal-fueled plants without CCS, then 82097 tons of $\mathrm{CO}_{2}$ will be produced in a whole day. Even with the wind power assisted, the $\mathrm{CO}_{2}$ emission will reach at least 70737 tons. However, with the help of $\mathrm{CCS}$, the $\mathrm{CO}_{2}$ produced can be further cut down to 32449 tons, as demonstrated in Fig. 4.

\subsection{Advantages of flexible CCS}

The CCS devices can swiftly reduce their power output to provide more available margin for upward spinning reserves, as shown in Fig. 5.

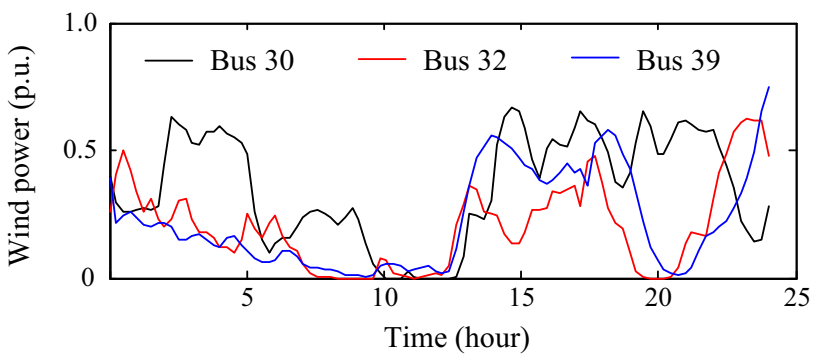

(a) Wind power forecast

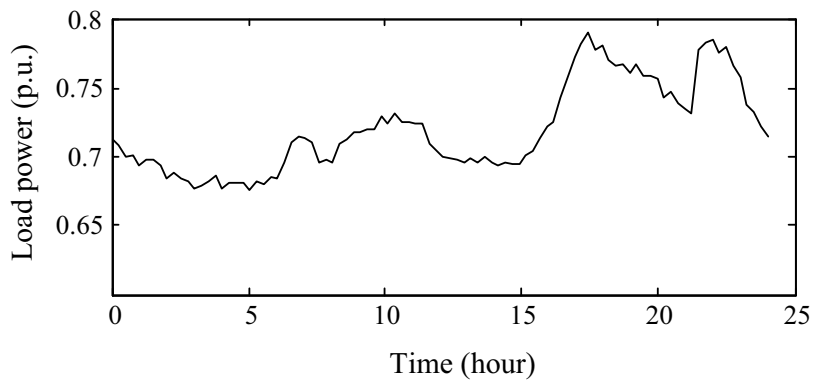

(b) Load forecast

Fig. 1 Load and wind farm output forecasts

Table 1 CCS data in the base case

\begin{tabular}{|c|c|c|c|c|c|c|c|c|c|}
\hline \multirow[t]{2}{*}{ Bus number } & \multirow{2}{*}{$\begin{array}{l}P_{i \mathrm{FIX}}^{\mathrm{CCS}} \\
(\mathrm{MW})\end{array}$} & \multicolumn{2}{|c|}{$\mathrm{CO}_{2}$ capture rate } & \multirow{2}{*}{$\begin{array}{l}\lambda_{i}^{\mathrm{GE}} \\
\left(\mathrm{MWh} / \mathrm{tCO}_{2}\right)\end{array}$} & \multirow{2}{*}{$\begin{array}{l}e_{i}^{\mathrm{G}} \\
\left(\mathrm{tCO}_{2} / \mathrm{MWh}\right)\end{array}$} & \multicolumn{4}{|c|}{15 min ramp range (MW) } \\
\hline & & $\beta_{i \text { max }}^{\mathrm{CCS}}$ & $\beta_{i \text { min }}^{\mathrm{CCS}}$ & & & $U_{i}^{\mathrm{CCS}}$ & $D_{i}^{\mathrm{CCS}}$ & $P_{i \mathrm{SU}}^{\mathrm{CCS}}$ & $P_{i \mathrm{SD}}^{\mathrm{CCS}}$ \\
\hline 30 & 5.2 & 0.9 & 0 & 0.23 & 0.76 & 122 & 122 & 120 & 120 \\
\hline 31 & 3.23 & 0.9 & 0 & 0.23 & 0.76 & 76 & 76 & 75 & 75 \\
\hline 32 & 3.62 & 0.9 & 0 & 0.23 & 0.76 & 85 & 85 & 85 & 85 \\
\hline 33 & 3.26 & 0.9 & 0 & 0.23 & 0.76 & 76 & 76 & 75 & 75 \\
\hline 39 & 5.5 & 0.9 & 0 & 0.23 & 0.76 & 130 & 130 & 130 & 130 \\
\hline
\end{tabular}

Table 2 Generator data in the base case

\begin{tabular}{|c|c|c|c|c|c|c|c|c|c|c|c|c|c|}
\hline \multirow[t]{2}{*}{ Bus number } & \multicolumn{2}{|c|}{$\begin{array}{l}\text { Technical limits } \\
\text { (MW) }\end{array}$} & \multicolumn{4}{|c|}{15 min ramp range (MW) } & \multicolumn{2}{|c|}{$\begin{array}{l}\text { Up and down time } \\
\text { (hour) }\end{array}$} & \multicolumn{3}{|c|}{$\begin{array}{l}\text { Fuel cost coefficients }(\$ / \\
\text { MWh) }\end{array}$} & \multicolumn{2}{|c|}{$\begin{array}{l}\text { Startup/shut down } \\
\text { cost }(\$)\end{array}$} \\
\hline & $P_{i \max }^{\mathrm{Coal}}$ & $P_{i \min }^{\text {Coal }}$ & $U_{i}^{\text {Coal }}$ & $D_{i}^{\text {Coal }}$ & $P_{i \mathrm{SU}}^{\mathrm{Coal}}$ & $P_{i \mathrm{SD}}^{\text {Coal }}$ & $T_{i \mathrm{UP}}$ & $T_{i \mathrm{DN}}$ & $a$ & $b$ & $c$ & $A_{i}^{\mathrm{SU}}$ & $A_{i}^{\mathrm{SD}}$ \\
\hline 30 & 1040 & 416.0 & 156 & 156 & 420 & 420 & 10.0 & 9.0 & 0.0140 & 20 & 500 & 7000 & 7000 \\
\hline 31 & 646 & 258.4 & 120 & 120 & 270 & 270 & 7.5 & 6.0 & 0.0200 & 20 & 380 & 5500 & 5500 \\
\hline 32 & 725 & 290.0 & 130 & 130 & 300 & 300 & 8.5 & 7.5 & 0.0194 & 20 & 42 & 5500 & 5500 \\
\hline 33 & 652 & 260.8 & 110 & 110 & 270 & 270 & 7.0 & 7.0 & 0.0200 & 20 & 380 & 5000 & 5000 \\
\hline 34 & 508 & 203.2 & 80 & 80 & 210 & 210 & 6.0 & 6.0 & 0.0255 & 20 & 295 & 4000 & 4000 \\
\hline 35 & 687 & 274.8 & 105 & 105 & 280 & 280 & 7.5 & 7.5 & 0.0210 & 20 & 400 & 5500 & 5500 \\
\hline 36 & 580 & 232.0 & 90 & 90 & 240 & 240 & 6.5 & 6.0 & 0.0230 & 20 & 350 & 4500 & 4500 \\
\hline 37 & 564 & 225.6 & 90 & 90 & 230 & 230 & 6.5 & 6.0 & 0.0222 & 20 & 330 & 4500 & 4500 \\
\hline 38 & 865 & 364.0 & 150 & 150 & 370 & 370 & 9.5 & 9.0 & 0.0150 & 20 & 490 & 7000 & 7000 \\
\hline 39 & 1100 & 440.0 & 200 & 200 & 450 & 450 & 10.0 & 9.0 & 0.0140 & 20 & 500 & 7000 & 7000 \\
\hline
\end{tabular}




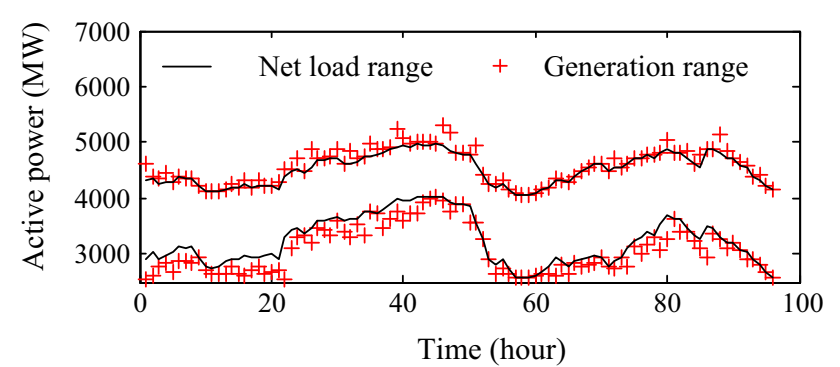

Fig. 2 Power balance between generation and net load

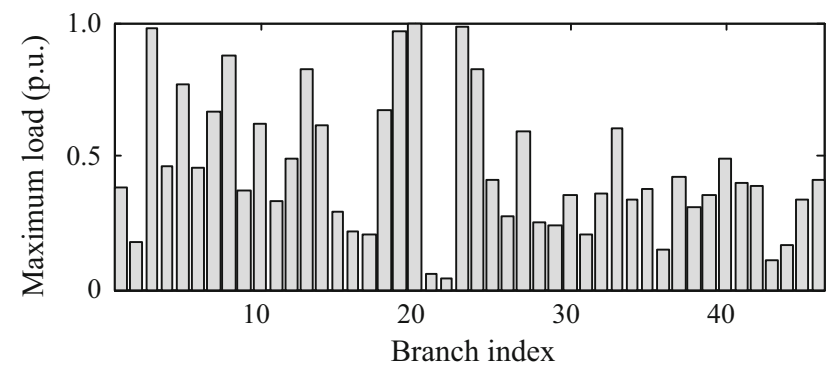

Fig. 3 Maximum load-carrying rate of the branches

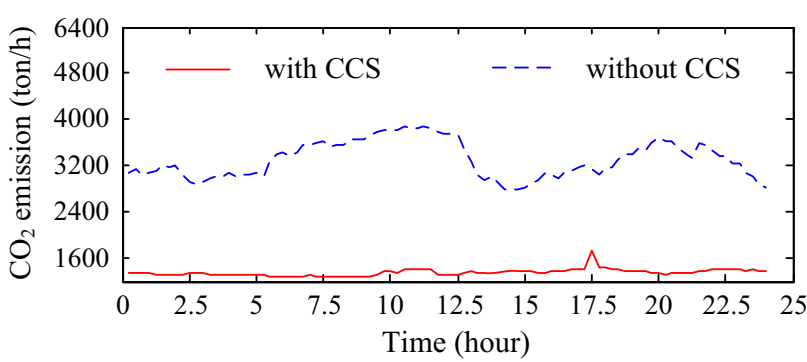

Fig. $4 \mathrm{CO}_{2}$ emission reduction

Furthermore, the CCS devices are allowed to operate at a derating status preparing to increase the power penalty when needed to provide extra downward spinning reserves.

However, the continuous period in derating operation of CCS devices will severely compromise the $\mathrm{CO}_{2}$ reduction objective. Hence, in the proposed LCUC model, the downward spinning reserve provided from CCS is optimized to be only an emergency precaution, as shown in Fig. 6 and Fig. 7.

\subsection{Controlling the robustness}

The robustness of the UC plan can be controlled by parameter $\Gamma_{t}^{\mathrm{W}}$ aforementioned, which can be chose from 0 (no uncertainty of wind power forecast is considered, namely the least robust) to the wind farm number (the whole wind power uncertainty intervals are considered, namely the most robust). The optimization objectives and

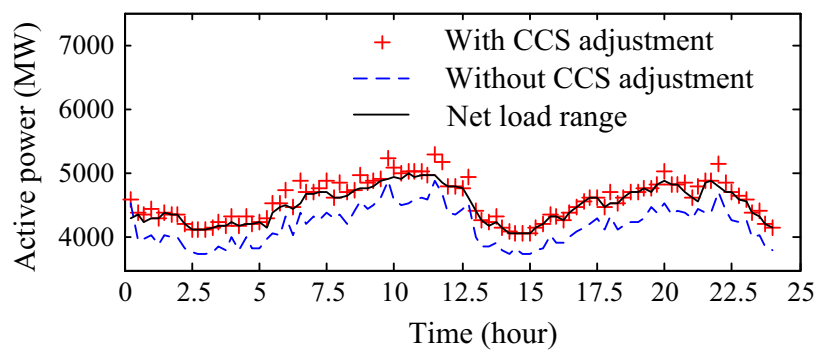

Fig. 5 Upward spinning reserve increased by CCS

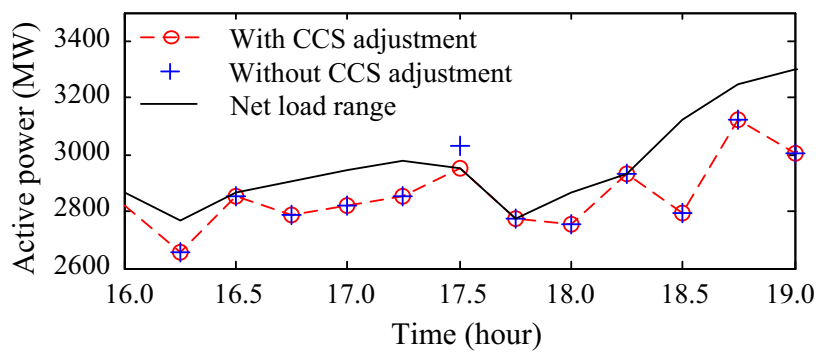

Fig. 6 Downward spinning reserve increased from 16:00 to 19:00

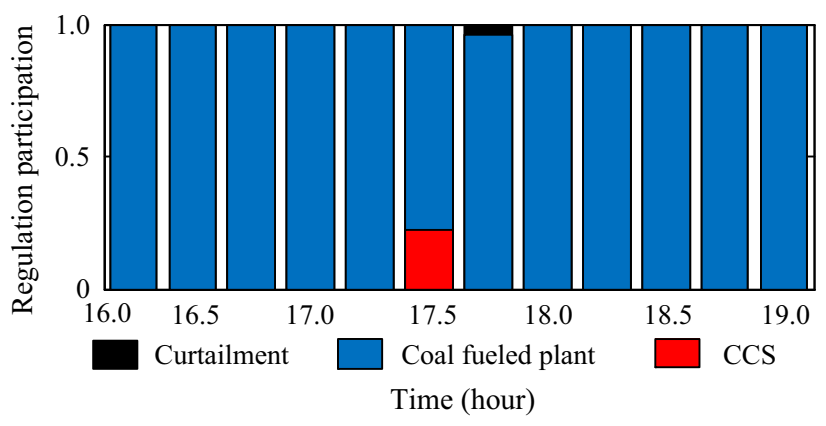

Fig. 7 Wind power regulation participation from 16:00 to 19:00

constraint violation probabilities with regard to different $\Gamma_{t}^{\mathrm{W}}$ are shown in Table 3.

It is shown that $\Gamma_{t}^{\mathrm{W}}$ provides a tradeoff between operation security and target optimality. With a smaller range of uncertainty considered, a more optimistic UC plan will be obtained. However the system operation situation may be more tend to risk emergency in the extreme scenarios. The results also indicate that calculation burden will increase when uncertainty of wind power is introduced. When $\Gamma_{t}^{\mathrm{W}}$ increases, the time required to solve the LCUC trends to increase.

\section{Conclusions}

A low-carbon unit commitment model considering flexible CCS and wind power forecast error uncertainties is proposed in this paper. The novel model is finally 
Table 3 Tradeoff between operation price and robustness

\begin{tabular}{lllll}
\hline$\Gamma_{t}^{\mathrm{W}}$ & Total cost $(\$)$ & $\mathrm{CO}_{2}$ emission (tons) & Violation probability & Calculation time (s) \\
\hline 3 & 4081292.5 & 32449.3 & $0 \%$ & 600.29 \\
2.5 & 4075222.8 & 32119.8 & $27.7 \%$ & 244.42 \\
2.2 & 4071959.8 & 31969.4 & $71.64 \%$ & 176.91 \\
2.1 & 4070902.9 & 31919.3 & $83.31 \%$ & 225.55 \\
2 & 4069851.7 & 31869.2 & $92.34 \%$ & 142.26 \\
1.5 & 4062824.9 & 31414.3 & $100 \%$ & 149.78 \\
0 & 4042371.0 & 29375.8 & $100 \%$ & 3.43 \\
\hline
\end{tabular}

converted into a deterministic MILP problem for solution, with a proposed linear re-dispatch strategy and application of linear robust optimization as well. Case studies in New England 39-bus system indicate that the model can efficiently provide reliable and low-carbon UC as well as redispatch plan against all realizations of wind power scenarios. Moreover, the robustness of the UC plan can be adjusted to avoid the conservatism which is an attractive feature in practical applications.

Further discussion shows that CCS technique is favorable for intensive wind power accommodation by increasing equivalent ramping rate of generators leading to more adequate spinning reserve. However its adverse effect on constantly changing the $\mathrm{CO}_{2}$ absorption rate must be carefully considered.

Acknowledgment This work was supported by the National Basic Research Program of China (No. 2012CB215106) and State Grid Corporation of China (No. 52150014006W).

Open Access This article is distributed under the terms of the Creative Commons Attribution License which permits any use, distribution, and reproduction in any medium, provided the original author(s) and the source are credited.

\section{References}

[1] Stocker TF, Qin D, Plattner GK et al (2014) Climate change 2013: the physical science basis. IPCC, Geneva

[2] Bernstein L, Bosch P, Canziani O et al (2008) Climate change 2007: synthesis report. IPCC, Geneva

[3] Metz B, Davidson O, de Coninck HC et al (2005) IPCC special report on carbon dioxide capture and storage. Cambridge University Press, Cambridge

[4] Martens P, Delarue E, D'haeseleer W (2012) A mixed integer linear programming model for a pulverized coal plant with post-combustion carbon capture. IEEE Trans Power Syst 27(2):741-751

[5] Ziaii S, Cohen S, Rochelle GT et al (2009) Dynamic operation of amine scrubbing in response to electricity demand and pricing. Energy Proc 1(1):4047-4053

[6] Chen QX, Kang CQ, Xia Q (2010) Modeling flexible operation mechanism of $\mathrm{CO}_{2}$ capture power plant and its effects on powersystem operation. IEEE Trans Energy Convers 25(3):853-861

[7] Chen QX, Kang CQ, Xia Q et al (2012) Optimal flexible operation of a $\mathrm{CO}_{2}$ capture power plant in a combined energy and carbon emission market. IEEE Trans Power Syst 27(3): 1602-1609
[8] Lu SY, Lou SH, Wu YW et al (2013) Power system economic dispatch under low-carbon economy with carbon capture plants considered. IET Gener Transm Distrib 7(9):991-1001

[9] Zhang Y, Wang JX, Wang XF (2014) Review on probabilistic forecasting of wind power generation. Renew Sustain Energy Rev 32:255-270

[10] Lee TY (2007) Optimal spinning reserve for a wind-thermal power system using EIPSO. IEEE Trans Power Syst 22(4):1612-1621

[11] Chaiyabut N, Damrongkulkumjorn P (2014) Optimal spinning reserve for wind power uncertainty by unit commitment with EENS constraint. In: Proceedings of the 2014 IEEE PES innovative smart grid technologies conference (ISGT'14), Washington, 19-22 Feb 2014, 5 pp

[12] Pappala VS, Erlich I, Rohrig K et al (2009) A stochastic model for the optimal operation of a wind-thermal power system. IEEE Trans Power Syst 24(2):940-950

[13] Wang JH, Shahidehpour M, Li ZY (2008) Security-constrained unit commitment with volatile wind power generation. IEEE Trans Power Syst 23(3):1319-1327

[14] Zhao CY, Wang JH, Watson JP et al (2013) Multi-stage robust unit commitment considering wind and demand response uncertainties. IEEE Trans Power Syst 28(3):2708-2717

[15] Wang Y, Xia Q, Kang CQ (2011) Unit commitment with volatile node injections by using interval optimization. IEEE Trans Power Syst 26(3):1705-1713

[16] Wang QF, Guan YP, Wang JH (2012) A chance-constrained two-stage stochastic program for unit commitment with uncertain wind power output. IEEE Trans Power Syst 27(1):206-215

[17] Chalmers H, Gibbins J (2006) Potential for synergy between renewables and carbon capture and storage. In: Proceedings of the 29th IAEE international conference, Potsdam, 7-10 Jun 2006, $16 \mathrm{pp}$

[18] van der Wijk PC, Brouwer AS, van den Broek M et al (2014) Benefits of coal-fired power generation with flexible CCS in a future northwest European power system with large scale wind power. Int J Greenhouse Gas Contrib 28:216-233

[19] Ji Z, Kang CQ, Chen QX et al (2013) Low-carbon power system dispatch incorporating carbon capture power plants. IEEE Trans Power Syst 28(4):4615-4623

[20] Yamashita D, Niimura T, Yokoyama R et al (2011) Thermal unit scheduling for $\mathrm{CO}_{2}$ reduction including significant wind power penetration. In: Proceedings of the 2011 IEEE Power and Energy Society general meeting, San Diego, 24-29 Jul 2011, 5 pp

[21] Carrión M, Arroyo JM (2006) A computationally efficient mixed-integer linear formulation for the thermal unit commitment problem. IEEE Trans Power Syst 21(3):1371-1378

[22] Bertsimas D, Sim M (2004) The price of robustness. Oper Res 52(1):35-53

[23] Kang SC (2008) Robust linear optimization using distributional information. Boston University, Boston 
[24] Zimmerman RD, Murillo-Sanchez CE (1997) MATPOWER: a MATLABTM power system simulation package, version 2.0, user's manual. Cornell University, Ithaca

Jiaming LI received the B.Eng. degree in electrical engineering from Huazhong University of Science and Technology (HUST) in 2012. He is now a Ph.D. candidate in HUST. His research interests include power system restoration after cascading failure and power system operation with significant renewable energy.

Jinyu WEN received the B.Eng. and Ph.D. degrees in electrical engineering from Huazhong University of Science and Technology (HUST), Wuhan, China, in 1992 and 1998, respectively. He was a visiting student from 1996 to 1997 and research fellow from 2002 to 2003 at the University of Liverpool, UK, and a senior visiting researcher at the University of Texas at Arlington, USA in 2010. From 1998 to 2002 he was a director engineer in XJ Electric Co. Ltd. in China. In 2003 he joined the HUST and now is a professor at HUST. His current research interests include renewable energy integration, energy storage application, DC grid, and power system operation and control.

Xingning HAN received the B.Eng. degree in electrical engineering from Hohai University, Nanjing, China, in 2011. Currently, she is pursuing the Ph.D. degree in electrical and electronic engineering from Huazhong University of Science and Technology (HUST), Wuhan, China. Her current research interest is the optimization with energy storage system. 